\title{
INTEGRATED FRACTIONAL WHITE NOISE AS AN ALTERNATIVE TO MULTIFRACTIONAL BROWNIAN MOTION
}

\author{
ALLAN SLY, ${ }^{*}$ University of California
}

\begin{abstract}
Multifractional Brownian motion is a Gaussian process which has changing scaling properties generated by varying the local Hölder exponent. We show that multifractional Brownian motion is very sensitive to changes in the selected Hölder exponent and has extreme changes in magnitude. We suggest an alternative stochastic process, called integrated fractional white noise, which retains the important local properties but avoids the undesirable oscillations in magnitude. We also show how the Hölder exponent can be estimated locally from discrete data in this model.
\end{abstract}

Keywords: Gaussian process; fractional Brownian motion; multifractional Brownian motion; Hölder exponent; identification

2000 Mathematics Subject Classification: Primary 60G18

Secondary 60G15

\section{Introduction. Problems with the multifractional Brownian motion formulation}

Motivation for work on processes with nonconstant scaling comes from the growing evidence for multiscaling/multifractal properties in applications. The general ideas were pioneered by Mandelbrot in a series of books and papers dating from the 1960s and now there are diverse applications from areas such as risky asset returns (see, e.g. [11] and [7]), fluid turbulence (see, e.g. [14]), geomagnetic time series (see, e.g. [1] and [16] and references therein), phylogenetic trees and genome sequencing (see, e.g. [17]), and telecommunications modelling (see, e.g. [12]).

Multifractal models are generally not tractable beyond the descriptive level, but scaling functions are commonly piecewise linear, indeed often bilinear. This offers the possibility of modelling with a small number of scales, possibly just two. If the underlying distribution can be treated as Gaussian then considerable explicit behavioural information can potentially be obtained.

Multifractional Brownian motion (MBM) was developed in order to model processes where the local roughness varies. The roughness is a local scaling property which is measured by the local Hölder exponent (see (2.6)). MBM was introduced by Peltier and Lévy Véhel [13], based on the integral moving average representation of fractional Brownian motion

$$
M_{H}(t)=\frac{1}{\Gamma\left(H(t)+\frac{1}{2}\right)} \int_{-\infty}^{t}(t-u)^{H(t)-1 / 2} W(\mathrm{~d} u)-\int_{-\infty}^{0}(-u)^{H(t)-1 / 2} W(\mathrm{~d} u),
$$

where $W$ is a Wiener measure and $H(t)$ is the local Hurst parameter. A common variation, the harmonizable integral representation version of MBM, which Stoev and Taqqu [15] showed is

Received 4 September 2006; revision received 22 January 2007.

* Postal address: Department of Statistics, University of California, 367 Evans Hall, Berkeley, CA 94720-3860, USA.

Email address: allansly@gmail.com 


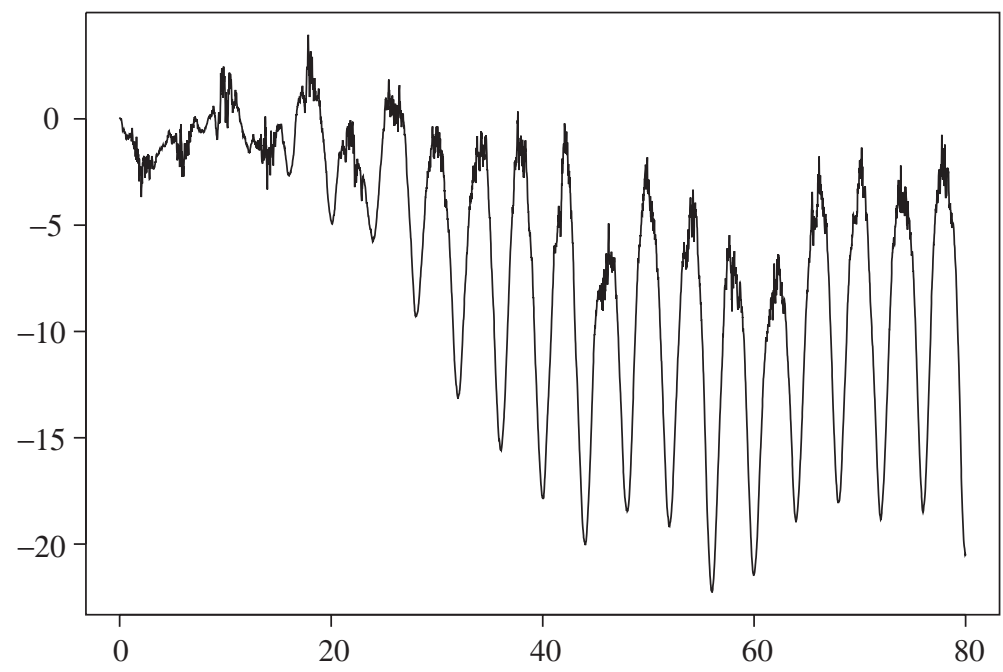

Figure 1: Multifractional Brownian motion with $H(t)=\frac{1}{2}+\frac{1}{4} \cos (\pi t / 2)$.

a different process, is given by

$$
M_{H}(t)=\operatorname{Re} \int_{\mathbb{R}} \frac{\mathrm{e}^{\mathrm{i} \xi t}-1}{|\xi|^{H(t)+1 / 2}} \tilde{W}(\mathrm{~d} \xi),
$$

where $\tilde{W}=W_{1}+\mathrm{i} W_{2}$ and $W_{1}$ and $W_{2}$ are independent Wiener processes. Our process will be based on the harmonizable representation. When $H(t)$ is constant, MBM is simply fractional Brownian motion. Both of these definitions can be written $M_{H}(t):=B_{H(t)}(t)$, where $B_{H}(t)$ is a family of fractional Brownian motions which is continuous in both $t$ and $H$. While there is only one fractional Brownian motion for each $H$, Stoev and Taqqu [15] showed that there is a large class of families of MBMs with nontrivially different covariance structures.

The definition of MBM allows the Hölder exponent to be specified at each point in time, meaning that the Hölder exponent of $M_{H}(t)$ is $H(t)$ almost surely. The processes are also locally asymptotically self-similar. A process is locally asymptotically self-similar at $t$ with parameter $H$ if

$$
\frac{M_{H}(t+s h)-M_{H}(t)}{h^{H}} \stackrel{\mathrm{D}}{\rightarrow} V(s),
$$

where $V(s)$ is the self-similar tangent process. The tangent process for MBM is fractional Brownian motion. Variations on MBM have been proposed in order to expand the class of functions $H(t)$ on which it can be defined (see, e.g. [2] and [5]).

When MBM has a nonconstant $H(t)$, it follows from the local self-similarity property that it does not have stationary increments. This is unavoidable, as varying roughness implies that the distribution of increments varies in time. However, the increment $M_{H}(t+s)-M_{H}(t)$ depends not just on $H(t)$ and $H(t+s)$ but also on $t$ itself. Regardless of the family of fraction Brownian motions used, by the triangle inequality

$$
\mathrm{E}\left(M_{H}(t+s)-M_{H}(t)\right)^{2} \geq\left((t+s)^{H(t+s)}-t^{H}\right)^{2}
$$


and, as such, when $t$ is large with $s$ fixed, small changes in $H(t)$ lead to very large increments. For example, if $t=100$ and $H(t)=H(t+1)=0.75$, then $\mathrm{E}\left(M_{H}(t+1)-M_{H}(t)\right)^{2}=1$, but if instead $H(t+1)=0.8$, then $\mathrm{E}\left(M_{H}(t+1)-M_{H}(t)\right)^{2}>63$. If $t=1000$ then $\mathrm{E}\left(M_{H}(t+1)-M_{H}(t)\right)^{2}>5411$. Figure 1 shows a typical sample path of MBM with $H(t)=$ $\frac{1}{2}+\frac{1}{4} \cos (\pi t / 2)$. All the figures in this paper were simulated using a simple discretization of the stochastic integral. As $t$ increases the difference between $B_{0.75}(t)$ and $B_{0.25}(t)$ increases and the magnitude of the oscillations increases accordingly. While the purpose of MBM is to vary the local fractal properties, unnecessary fluctuations and oscillations are introduced into the process.

In this paper we introduce a new class of Gaussian processes, called integrated fractional white noise, which avoids these problems while retaining the essential Hölder exponent and local asymptotic self-similarity properties of MBM. The variances of the increments $M_{H}(t+s)$ $-M_{H}(t)$ depend only on $s$ and the values taken by $H$ between $t$ and $t+s$, and not on $t$ itself. This avoids the sudden swings in magnitude and wild oscillations associated with changing values of the Hurst parameter. Retaining the essential features of MBM while more plausible trajectories makes integrated fractional white noise a more reasonable model.

In Section 2 we define the process and establish its key properties. In Section 3 it is shown to be identifiable by constructing a strongly consistent estimator of the process. This is a significant improvement over earlier estimators of MBM. Using a spectral decomposition we establish the asymptotic normality of the estimator under mild conditions. Some proofs are postponed to Section 4.

\section{Integrated fractional white noise}

To motivate the definition of integrated fractional white noise, we will break MBM into two parts. While MBM is obviously not differentiable, it can be differentiated as a stochastic process in the space of stochastic distributions. Assuming that $H(t)$ is continuously differentiable,

$$
\frac{\mathrm{d}}{\mathrm{d} t} M_{H}(t)=H^{\prime}(t) \frac{\partial}{\partial H} B_{H}(t)+W_{H}(t)
$$

where $W_{H}(t)$ is fractional white noise, the derivative of fractional Brownian motion in the space of stochastic distributions, as in [9]. Unlike fractional white noise, the term $(\partial / \partial H) B_{H}(t)$ is a Gaussian random variable with variance bounded on compacts. It becomes very large when $t$ is large, since the difference between $B_{H_{1}}(t)$ and $B_{H_{2}}(t)$ becomes very large. Then

$$
\begin{aligned}
M_{H}^{(f)}(t) & :=\int_{0}^{t} H^{\prime}(s) \frac{\partial}{\partial H} B_{H}(s) \mathrm{d} s \\
& =\int_{0}^{t} H^{\prime}(s) \operatorname{Re} \int_{\mathbb{R}} \frac{\ln (|\xi|)\left(\mathrm{e}^{\mathrm{i} \xi t}-1\right)}{|\xi|^{H(t)+1 / 2}} \tilde{W}(\mathrm{~d} \xi) \mathrm{d} s
\end{aligned}
$$

is a Gaussian finite variation process with locally Lipschitz paths. It follows that $M_{H}(t)-$ $M_{H}^{(f)}(t)$ is also locally asymptotically self-similar and has local Hölder function $H(t)$, and we take the following as our definition.

Definition 2.1. (Integrated fractional white noise.) For $0<H(t)<1$ and $H(t)$ continuous, we define integrated fractional white noise as

$$
Y_{H}(t)=\operatorname{Re} \int_{\mathbb{R}} \int_{0}^{t} \frac{\mathrm{i} \xi \mathrm{e}^{\mathrm{i} \xi s}}{|\xi|^{H(s)+1 / 2}} \mathrm{~d} s \tilde{W}(\mathrm{~d} \xi),
$$

given that the integrand is in $L^{2}(\mathbb{R})$. 
This definition is equivalent to

$$
Y_{H}(t)=\int_{0}^{t} W_{H(s)}(s) \mathrm{d} s
$$

see Appendix A for proof. When $H(t)$ is less than $\frac{1}{2}$ this stochastic distribution may not be in $L^{2}$ and greater regularity of $H(t)$ must therefore be assumed.

Theorem 2.1. Suppose that $H(t)$ is continuous and that $0<a \leq H(t) \leq b<1$. Assume that there exist some $\beta, C_{1}>0$ such that

$$
\beta+a>\frac{1}{2}
$$

and

$$
|H(t)-H(s)| \leq C_{1}|t-s|^{\beta} .
$$

Then $Y_{H}(t) \in L^{2}$ with

$$
\begin{aligned}
\mathrm{E} Y_{H}(t)^{2}= & \int_{0}^{t} A(H(s)) H(s) s^{2 H(s)-1} \mathrm{~d} s+\int_{0}^{t} A(H(s)) H(s)(t-s)^{2 H(s)-1} \mathrm{~d} s \\
& +\frac{1}{4} \int_{0}^{t} \int_{0}^{t} f_{H}(x, y) \mathrm{d} x \mathrm{~d} y \\
< & \infty
\end{aligned}
$$

where

$$
A(H)=\int_{\mathbb{R}}\left|\int_{0}^{1} \frac{\mathrm{i} \xi \mathrm{e}^{\mathrm{i} \xi s}}{|\xi|^{H+1 / 2}} \mathrm{~d} s\right|^{2} \mathrm{~d} \xi=\frac{\pi}{H \Gamma(2 H) \sin (H \pi)}
$$

and

$$
\begin{aligned}
f_{H}(x, y)= & 2 A\left(\frac{H(x, y)}{2}\right) H(x, y)(H(x, y)-1)|x-y|^{H(x, y)-2} \\
& -A(H(x)) 2 H(x)(2 H(x)-1)|x-y|^{2 H(x)-2} \\
& -A(H(y)) 2 H(y)(2 H(y)-1)|x-y|^{2 H(y)-2}
\end{aligned}
$$

with $H(x, y)=H(x)+H(y)$. The process shares the relevant important local properties with multifractional Brownian motion: $Y_{H}(t)$ is locally asymptotically self-similar, since

$$
h^{-H\left(t_{0}\right)}\left(Y_{H}\left(t_{0}+t h\right)-Y_{H}\left(t_{0}\right)\right) \stackrel{\mathrm{D}}{\rightarrow} A\left(H\left(t_{0}\right)\right)^{1 / 2} B_{H\left(t_{0}\right)}(t)
$$

as $h \rightarrow 0$, where convergence is in finite-dimensional distributions and the tangent process $B_{H\left(t_{0}\right)}(t)$ is fractional Brownian motion with parameter $H\left(t_{0}\right)$; and, with probability $1, Y_{H}(t)$ has continuous paths with Hölder exponent $H(t)$ at $t$, that is,

$$
\sup \left\{\gamma: \lim _{h \rightarrow 0}|h|^{-\gamma}\left|Y_{H}(t+h)-Y_{H}(t)\right|=0\right\}=H(t) .
$$

See Appendix A for proof of Theorem 2.1.

Figure 2 shows a typical sample path of $Y_{H}(t)$ with $H(t)=\frac{1}{2}+\frac{1}{4} \cos (\pi t / 2)$. It exhibits areas of high and low roughness because of the varying local Hurst parameter, but there are no extremely large increments or wild oscillations like those in Figure 1. The difference is that the distribution of the increments of integrated fractional white noise depends only on the local 


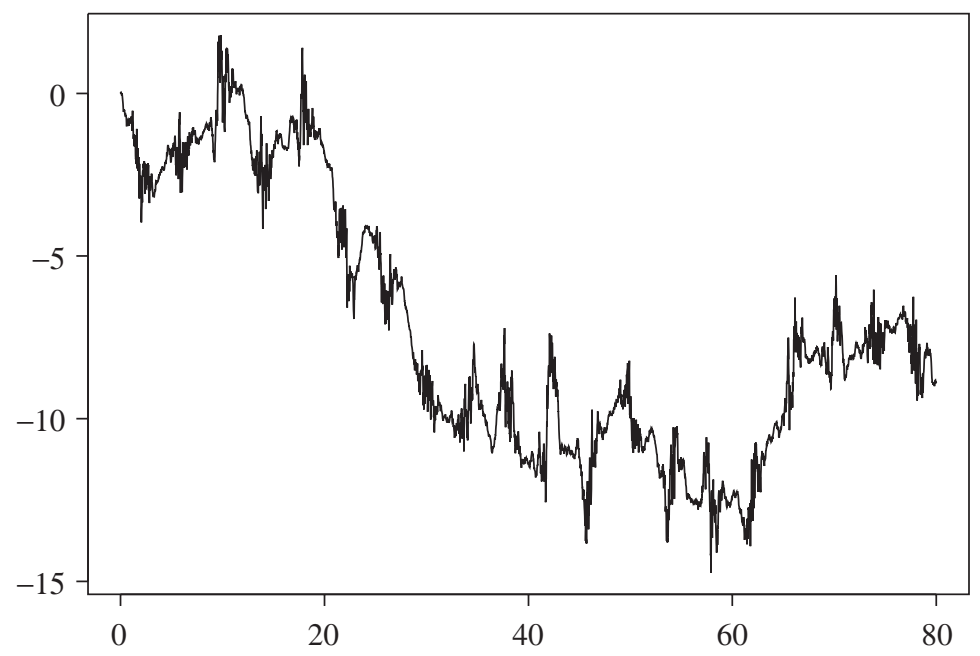

Figure 2: $Y_{H}(t)$ with $H(t)=\frac{1}{2}+\frac{1}{10} \cos (5 \pi t)$.

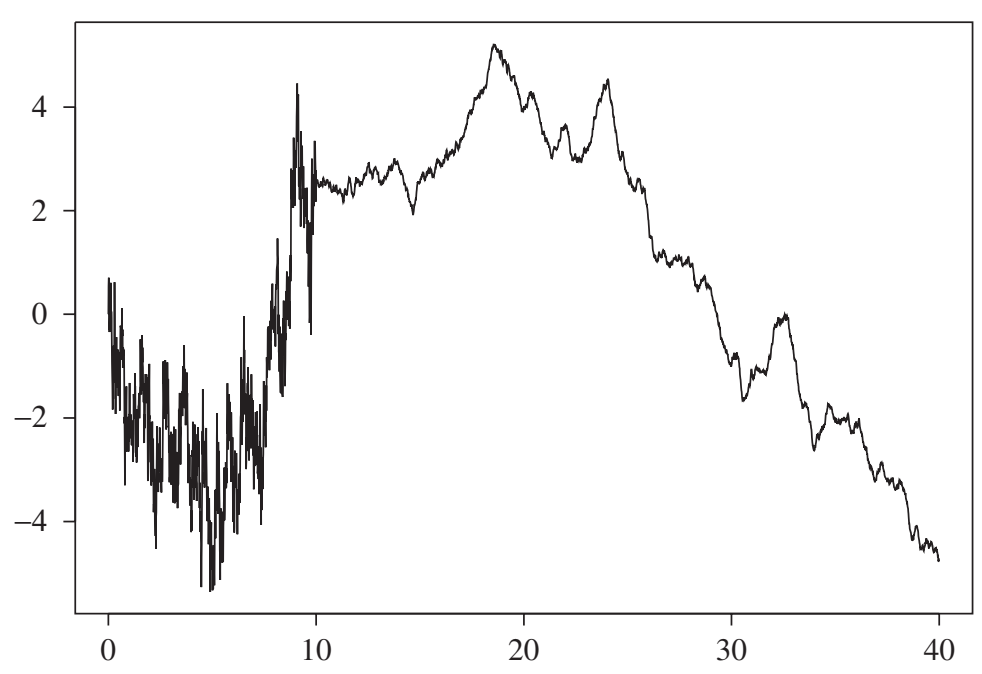

Figure 3: $Y_{H}(t)$ with discontinuous $H(t)$.

values of $H(t)$ and not on its position in time, in the following sense: if $H_{1}(t)=H_{2}(t+u)$ for all $t \in[a, b]$, then

$$
\left\{Y_{H_{1}}(a+t)-Y_{H_{1}}(a)\right\}_{t \in[0, b-a]} \stackrel{\mathrm{D}}{=}\left\{Y_{H_{2}}(a+u+t)-Y_{H_{2}}(a+u)\right\}_{t \in[0, b-a]}
$$

in the sense of finite-dimensional distributions. As a result, the variance of the increments can be calculated using (2.7). This property of (2.5) is also important for the generalization when $H(t)$ is random. Suppose that $H(t)$ is a stationary process whose paths satisfy conditions (2.3) and (2.4). Then it follows that $Y_{H}(t)$ has stationary increments. 
The definition of $Y_{H}(t)$ also naturally extends to piecewise-continuous functions. Unlike for MBM, this does not lead to discontinuities. Figure 3 shows a typical sample path of $Y_{H}(t)$ with

$$
H(t)= \begin{cases}\frac{1}{4}, & t \leq 10 \\ \frac{3}{4}, & t>10 .\end{cases}
$$

Throughout the rest of this paper we will assume that $H(t)$ satisfies conditions $(2.3)$ and (2.4).

\section{Identification of $H(t)$}

For processes like fractional Brownian motion where the Hölder exponent is constant, it can be estimated by examining either local or long-range properties. For every type of MBM, the Hölder exponent is a truly local property and must be estimated as such. The estimator used most frequently in the MBM literature (see, e.g. [2], [3], and [6]) is

$$
\hat{H}_{N}(t)=\frac{1}{2}\left(1-\gamma-\frac{\ln V_{N}(t)}{\ln N}\right),
$$

where $0<\gamma<1$ and

$$
V_{N}(t)=\sum_{j=-\left\lfloor N^{1-\gamma}\right\rfloor}^{\left\lfloor N^{1-\gamma}\right\rfloor}\left(Y_{H}\left(t+\frac{j+1}{N}\right)-2 Y_{H}\left(t+\frac{j}{N}\right)+Y_{H}\left(t+\frac{j-1}{N}\right)\right)^{2} .
$$

While this estimator is strongly consistent, it does not converge very quickly. Heuristically E $V_{N}(t) \approx C(t) N^{1-\gamma-2 H}$, so

$$
\frac{\ln V_{N}(t)}{\ln N} \approx \frac{\ln C(t)}{\ln N}+1-\gamma-2 H .
$$

For $\varepsilon_{n}=\hat{H}_{N}(t)-H(t)+\ln C(t) /(2 \ln N)$, if $(1-\gamma)(4 H(t)-3)-4 \beta<0$ then $c N^{(1-\gamma) / 2} \varepsilon_{n}$ converges in distribution to $N(0,1)$, by a modification of the proof of Theorem 3.2 (see below). However, the term $\ln C(t) /(2 \ln N)$ depends on $H(t)$ and decays very slowly to 0 , making $\hat{H}_{N}(t)$ a very inefficient estimator of $H(t)$. We can prove better rate-of-convergence results for the estimator

$$
\breve{H}_{N}(t)=\frac{1}{2}\left(\log _{2} \frac{1+2\left\lfloor N^{1-\gamma}\right\rfloor}{1+2\left\lfloor(N / 2)^{1-\gamma}\right\rfloor}+\log _{2} \frac{V_{N / 2}(t)}{V_{N}(t)}\right) .
$$

This estimator was used in [4], albeit for a different process. We will prove consistency and a central limit theorem for this estimator, but first some lemmas are required.

Lemma 3.1. Let $X_{j}=Y_{H}(t+j / N)-Y_{H}(t+(j-1) / N)$. Then there exist constants $C_{3}, C_{4}, C_{5}, C_{6}>0$ such that, for all $N>1$ and $i$ and $j$ with $-\left\lfloor N^{1-\gamma}\right\rfloor \leq i, j \leq\left\lfloor N^{1-\gamma}\right\rfloor$,

$$
\left|\mathrm{E}\left(X_{i+1}-X_{i}\right)^{2}-\left(4-4^{H(t)}\right) A(H(t)) N^{-2 H(t)}\right| \leq C_{3} \ln (N) N^{-2 H(t)-\gamma \beta} .
$$

Furthermore,

$$
\begin{aligned}
& \mid \mathrm{E}\left(X_{i+1}-X_{i}\right)\left(X_{j+1}-X_{j}\right)-\frac{1}{2}\left(-|i-j+2|^{2 H(t)}+4|i-j+1|^{2 H(t)}\right. \\
& \left.-6|i-j|^{2 H(t)}+4|i-j-1|^{2 H(t)}-|i-j-2|^{2 H(t)}\right) A(H(t)) N^{-2 H(t)} \mid \\
& \quad \leq C_{4}|i-j|^{2 H(t)-2} \ln (N)^{2} N^{-2 H(t)-2 \beta}+C_{5}|i-j|^{2 H(t)-3} \ln (N)^{2} N^{-2 H(t)-\gamma \beta} .
\end{aligned}
$$


Also,

$$
\begin{aligned}
& \frac{1}{2} A(H(t))|-| i-j+\left.2\right|^{2 H(t)}+4|i-j+1|^{2 H(t)}-6|i-j|^{2 H(t)} \\
& \quad+4|i-j-1|^{2 H(t)}-|i-j-2|^{2 H(t)} \mid \\
& \leq C_{6}|i-j|^{2 H(t)-4} .
\end{aligned}
$$

See Appendix A for a proof of Lemma 3.1.

Corollary 3.1. There exist constants $C_{7}, C_{8}>0$ such that, for all $N$,

$$
C_{7} N^{1-\gamma-2 H(t)} \leq \mathrm{E} V_{N}(t) \leq C_{8} N^{1-\gamma-2 H(t)} .
$$

Lemma 3.2. There exists a constant $C_{9}>0$ such that, for all $N>1$,

$$
\mathrm{E}\left(V_{N}(t)-\mathrm{E} V_{N}(t)\right)^{2} \leq C_{9} N^{1-\gamma-4 H(t)}
$$

for $(1-\gamma)(4 H(t)-3)-4 \beta<0$ and

$$
\mathrm{E}\left(V_{N}(t)-\mathrm{E} V_{N}(t)\right)^{2} \leq C_{9} \ln (N)^{4} N^{1-\gamma-4 H(t)+(1-\gamma)(4 H(t)-3)-4 \beta}
$$

otherwise.

Proof. By Theorem 3.9 of [10],

$$
\operatorname{var} V_{N}(t)=2 \sum_{i=-\left\lfloor N^{1-\gamma}\right\rfloor}^{\left\lfloor N^{1-\gamma}\right\rfloor} \sum_{j=-\left\lfloor N^{1-\gamma}\right\rfloor}^{\left\lfloor N^{1-\gamma}\right\rfloor}\left(\mathrm{E}\left(X_{i+1}-X_{i}\right)\left(X_{j+1}-X_{j}\right)\right)^{2} .
$$

If $(1-\gamma)(4 H(t)-3)-4 \beta<0$ then, by Lemma 3.1,

$$
\begin{aligned}
& \sum_{j=-\left\lfloor N^{1-\gamma}\right\rfloor}^{\left\lfloor N^{1-\gamma}\right\rfloor+1}\left(\mathrm{E}\left(X_{i+1}-X_{i}\right)\left(X_{j+1}-X_{j}\right)\right)^{2} \\
& \leq\left(4-4^{-H}\right)^{2} A(H(t))^{2} N^{-4 H(t)}+C_{3}^{2} \ln (N)^{2} N^{-4 H(t)-2 \gamma \beta} \\
& \quad+12 N^{-4 H(t)} \sum_{j=1}^{\left\lfloor N^{1-\gamma}\right\rfloor} C_{4}|i-j|^{4 H(t)-4} \ln (N)^{4} N^{-2 H(t)-4 \beta} \\
& \quad+C_{5}|i-j|^{4 H(t)-6} \ln (N)^{4} N^{-2 \gamma \beta}+C_{6}|i-j|^{4 H(t)-8} \\
& \leq c_{1} N^{-4 H(t)}
\end{aligned}
$$

and, so,

$$
\mathrm{E}\left(V_{N}(t)-\mathrm{E} V_{N}(t)\right)^{2} \leq 2 \sum_{i=-\left\lfloor N^{1-\gamma}\right\rfloor}^{\left\lfloor N^{1-\gamma}\right\rfloor+1} c_{1} N^{-4 H(t)} \leq C_{9} N^{1-\gamma-4 H(t)} .
$$

Equation (3.3) follows similarly.

Theorem 3.1. Almost surely,

$$
\lim _{N \rightarrow \infty} \frac{V_{N}(t)}{\mathrm{E} V_{N}(t)}=1
$$

and, so, the estimator $\breve{H}_{N}(t)$ converges almost surely to $H(t)$ as $N \rightarrow \infty$. 
Proof. This result is an application of the Borel-Cantelli lemma. Let $\varepsilon>0$. By Lemma 3.2, there exists a $\delta$ such that $\left\|V_{N}(t)-\mathrm{E} V_{N}(t)\right\|_{2}^{2} \leq c N^{1-\gamma-4 H(t)+\delta}$ and $\delta<1-\gamma$. Then, by Lemma 3.1,

$$
\begin{aligned}
\mathrm{P}\left(\left|\frac{V_{N}(t)}{\mathrm{E} V_{N}(t)}-1\right|>\varepsilon\right) & =\mathrm{P}\left(\left|V_{N}(t)-\mathrm{E} V_{N}(t)\right|>\varepsilon \mathrm{E} V_{N}\right) \\
& \leq \mathrm{P}\left(\left|V_{N}(t)-\mathrm{E} V_{N}(t)\right|>\varepsilon \frac{C_{7}}{\sqrt{c}} N^{(1-\gamma-\delta) / 2}\left\|V_{N}(t)-\mathrm{E} V_{N}(t)\right\|_{2}\right)
\end{aligned}
$$

Since $V_{N}(t)-\mathrm{E} V_{N}(t)$ is a quadratic polynomial of Gaussian random variables, by Theorem 6.7 of [10], for $\varepsilon\left(C_{7} / \sqrt{c}\right) N^{(1-\gamma+\delta) / 2}>2$,

$$
\mathrm{P}\left(\left|V_{N}(t)-\mathrm{E} V_{N}(t)\right|>\varepsilon \frac{C_{7}}{\sqrt{c}} N^{(1-\gamma-\delta) / 2}\left\|V_{N}(t)-\mathrm{E} V_{N}(t)\right\|_{2}\right) \leq \exp \left(-\kappa \varepsilon \frac{C_{7}}{\sqrt{c}} N^{(1-\gamma-\delta) / 2}\right),
$$

where $\kappa>0$ is an absolute constant. This implies that

$$
\sum_{N=1}^{\infty} \mathrm{P}\left(\left|\frac{V_{N}(t)}{\mathrm{E} V_{N}(t)}-1\right|>\varepsilon\right)<\infty
$$

so the result follows from the Borel-Cantelli lemma. By Lemma 3.1,

$$
\lim _{N \rightarrow \infty} \log _{2} \frac{\mathrm{E} V_{N / 2}(t)}{\mathrm{E} V_{N}(t)}=-(1-\gamma)+2 H(t)
$$

hence,

$$
\lim _{N \rightarrow \infty} \frac{1}{2}\left(\log _{2} \frac{1+2\left\lfloor N^{1-\gamma}\right\rfloor}{1+2\left\lfloor(N / 2)^{1-\gamma}\right\rfloor}+\log _{2} \frac{V_{N / 2}(t)}{V_{N}(t)}\right)=H(t)
$$

almost surely.

It follows from Lemma 3.1 that, for $(1-\gamma)(4 H(t)-3)-4 \beta<0$,

$$
N^{-(1-\gamma)} E\left(\frac{V_{N}-\mathrm{E} V_{N}}{\mathrm{E} V_{N}}-\frac{V_{N / 2}-\mathrm{E} V_{N / 2}}{\mathrm{E} V_{N / 2}}\right)^{2} \rightarrow C_{10}
$$

where

$$
\begin{aligned}
C_{10}= & \frac{1+2^{-(1-\gamma)}}{4\left(4-4^{H}(t)\right)^{2}} \sum_{i=-\infty}^{\infty}\left(-|i+2|^{2 H(t)}+4|i+1|^{2 H(t)}-6|i|^{2 H(t)}\right. \\
& \left.+4|i-1|^{2 H(t)}-|i-2|^{2 H(t)}\right)^{2} \\
& +\frac{2^{1-\gamma}}{4\left(4-4^{H}(t)\right)^{2}} \sum_{i=-\infty}^{\infty}\left(-|i+3|^{2 H(t)}+2|i+2|^{2 H(t)}+|i+1|^{2 H(t)}\right. \\
& \left.\quad-4|i|^{2 H(t)}+|i-1|^{2 H(t)}+2|i-2|^{2 H(t)}-|i+3|^{2 H(t)}\right)^{2} .
\end{aligned}
$$


Lemma 3.3. There exists a constant $C_{11}>0$ such that, for all $N>1$,

$$
\left|2 H(t)-\log _{2} \frac{1+2\left\lfloor N^{1-\gamma}\right\rfloor}{1+2\left\lfloor(N / 2)^{1-\gamma}\right\rfloor}-\log _{2} \frac{\mathrm{E} V_{N / 2}}{\mathrm{E} V_{N}}\right| \leq C_{11} \ln (N) N^{-\gamma \beta} .
$$

Proof. Вy (3.1),

$$
\begin{aligned}
2 H(t)- & \log _{2} \frac{1+2\left\lfloor N^{1-\gamma}\right\rfloor}{1+2\left\lfloor(N / 2)^{1-\gamma}\right\rfloor}-\log _{2} \frac{\mathrm{E} V_{N / 2}}{\mathrm{E} V_{N}} \\
\leq & 2 H(t)-\log _{2}\left(\left(4-4^{H(t)}\right) A(H(t))(N / 2)^{-2 H(t)}\right. \\
& \left.-C_{3} \ln (N / 2)(N / 2)^{-2 H(t)-\gamma \beta}\right) \\
& +\log _{2}\left(\left(4-4^{H(t)}\right) A(H(t)) N^{-2 H(t)}+C_{3} \ln (N) N^{-2 H(t)-\gamma \beta}\right) \\
= & -\log _{2}\left(\left(4-4^{H(t)}\right) A(H(t))-C_{3} \ln (N / 2)(N / 2)^{-\gamma \beta}\right) \\
& +\log _{2}\left(\left(4-4^{H(t)}\right) A(H(t))+C_{3} \ln (N) N^{-\gamma \beta}\right) \\
\leq & C_{11} \ln (N) N^{-\gamma \beta} .
\end{aligned}
$$

The lower bound holds similarly, proving the result.

Lemma 3.4. For $(1-\gamma)(4 H(t)-3)-4 \beta<0$,

$$
\frac{1}{\sqrt{C_{10} N^{-(1-\gamma)}}}\left(\frac{V_{N}-\mathrm{E} V_{N}}{\mathrm{E} V_{N}}-\frac{V_{N / 2}-\mathrm{E} V_{N / 2}}{\mathrm{E} V_{N / 2}}\right) \stackrel{\mathrm{D}}{\rightarrow} N(0,1)
$$

as $N \rightarrow \infty$.

Proof. Let $H_{N}$ be the Gaussian Hilbert space generated by $\left\{X_{i+1}-X_{i}:-\left\lfloor N^{1-\gamma}\right\rfloor \leq i \leq\right.$ $\left.\left\lfloor N^{1-\gamma}\right\rfloor\right\}$. Then $\left(V_{N}-\mathrm{E} V_{N}\right) / \mathrm{E} V_{N}-\left(V_{N / 2}-\mathrm{E} V_{N / 2}\right) / \mathrm{E} V_{N / 2}$ is in the second homogeneous chaos of $H$ and, so, we apply the representation formula from Theorem 6.1 of [10]. Let $\tilde{T}_{N}: H_{N} \rightarrow H_{N}$ be the operator $\tilde{T}_{N}(\xi)=\frac{1}{2} \pi_{1}\left(\left(V_{N}-\mathrm{E} V_{N}\right) \xi\right)$ where $\pi_{1}$ is the orthogonal projection onto $H_{N}$. Acting on vectors of the form $\left(s_{i}\left(X_{i+1}-X_{i}\right)\right)_{-\left\lfloor N^{1-\gamma}\right\rfloor \leq i \leq\left\lfloor N^{1-\gamma}\right\rfloor}, \tilde{T}_{N}$ is the matrix

$$
\left[\mathrm{E}\left(X_{i+1}-X_{i}\right)\left(X_{j+1}-X_{j}\right)\right]_{i, j=-\left\lfloor N^{1-\gamma}\right\rfloor \cdots\left\lfloor N^{1-\gamma}\right\rfloor} .
$$

By a standard result in linear algebra, the largest eigenvalue of $\tilde{T}_{N}$ has absolute value at most

$$
\max _{i} \sum_{j=-\left\lfloor N^{1-\gamma}\right\rfloor}^{\left\lfloor N^{1-\gamma}\right\rfloor}\left|\mathrm{E}\left(X_{i+1}-X_{i}\right)\left(X_{j+1}-X_{j}\right)\right| .
$$

By Lemma 3.1, for $(2 H(t)-1)(1-\gamma)<2 \beta$,

$$
\begin{aligned}
& \sum_{j=-\left\lfloor N^{1-\gamma}\right\rfloor}^{\left\lfloor N^{1-\gamma}\right\rfloor}\left|\mathrm{E}\left(X_{i+1}-X_{i}\right)\left(X_{j+1}-X_{j}\right)\right| \\
& \quad \leq 2 \sum_{j=1}^{2\left\lfloor N^{1-\gamma}\right\rfloor} C_{4} j^{2 H(t)-2} \ln (N)^{2} N^{-2 H(t)-2 \beta} \\
& \quad+C_{5} j^{2 H(t)-3} \ln (N)^{2} N^{-2 H(t)-\gamma \beta}+C_{6} j^{2 H(t)-4} N^{-2 H(t)} \\
& \quad \leq c_{1} N^{-2 H(t)} .
\end{aligned}
$$


Now let $\tilde{T}_{N}^{\prime}$ be the operator

$$
\tilde{T}_{N}^{\prime}(\xi)=\frac{1}{2} \pi_{1}\left(\frac{1}{\sqrt{C_{10} N^{-(1-\gamma)}}}\left(\frac{V_{N}-\mathrm{E} V_{N}}{\mathrm{E} V_{N}}-\frac{V_{N / 2}-\mathrm{E} V_{N / 2}}{\mathrm{E} V_{N / 2}}\right) \xi\right) .
$$

Then, by Theorem 6.1 of [10],

$$
\frac{1}{\sqrt{C_{10} N^{-(1-\gamma)}}}\left(\frac{V_{N}-\mathrm{E} V_{N}}{\mathrm{E} V_{N}}-\frac{V_{N / 2}-\mathrm{E} V_{N / 2}}{\mathrm{E} V_{N / 2}}\right)
$$

can be rewritten as

$$
\sum_{j} \lambda_{j, N}\left(\xi_{j, N}^{2}-1\right)
$$

where the $\lambda_{j, N}$ are the eigenvalues of $\tilde{T}_{N}^{\prime}$ and (for fixed $N$ ) the $\xi_{j, N}$ are independent $N(0,1)$ distributed random variables. Since

$$
\tilde{T}_{N}^{\prime}=\frac{1}{\sqrt{C_{10} N^{-(1-\gamma)}}}\left(\frac{\tilde{T}_{N}}{\mathrm{E} V_{N}}-\frac{\tilde{T}_{N / 2}}{\mathrm{E} V_{N / 2}}\right),
$$

the maximum eigenvalue of $\tilde{T}_{N}^{\prime}$ is at most

$$
\frac{1}{\sqrt{C_{10} N^{-(1-\gamma)}}}\left(\frac{c_{1} N^{-2 H(t)}}{C_{7} N^{1-\gamma-2 H(t)}}+\frac{c_{1}(N / 2)^{-2 H(t)}}{C_{7}(N / 2)^{1-\gamma-2 H(t)}}\right) \leq c_{3} N^{-(1-\gamma) / 2}
$$

for $(2 H(t)-1)(1-\gamma)<2 \beta$, so $\max _{j}\left|\lambda_{j, N}\right| \rightarrow 0$ as $N \rightarrow \infty$. By Theorem 7.1.2 of [8],

$$
\frac{1}{\sqrt{C_{10} N^{-(1-\gamma)}}}\left(\frac{V_{N}-\mathrm{E} V_{N}}{\mathrm{E} V_{N}}-\frac{V_{N / 2}-\mathrm{E} V_{N / 2}}{\mathrm{E} V_{N / 2}}\right)=\sum_{j} \lambda_{j, N}\left(\xi_{j, N}^{2}-1\right) \stackrel{\mathrm{D}}{\rightarrow} N(0,1)
$$

as $N \rightarrow \infty$. Similar calculations hold for $(2 H(t)-1)(1-\gamma) \geq 2 \beta$.

Theorem 3.2. (Central limit theorem.) If $(1-\gamma)(4 H(t)-3)-4 \beta<0$ and

$$
\gamma>\frac{1}{1+2 \beta}
$$

then

$$
\frac{\ln 2}{\sqrt{C_{10} N^{-(1-\gamma)}}}\left(H(t)-\breve{H}_{N}(t)\right) \stackrel{\mathrm{D}}{\rightarrow} N(0,1)
$$

as $N \rightarrow \infty$, where $C_{10}$ is as given in (3.4).

Proof. By Lemma 3.3,

$$
\left|H(t)-\frac{1}{2}\left(\log _{2} \frac{1+2\left\lfloor N^{1-\gamma}\right\rfloor}{1+2\left\lfloor(N / 2)^{1-\gamma}\right\rfloor}+\log _{2} \frac{\mathrm{E} V_{N / 2}}{\mathrm{E} V_{N}}\right)\right| \leq C_{11} \ln (N) N^{-\gamma \beta}
$$

and, so,

$$
\frac{\ln 2}{\sqrt{C_{10} N^{-(1-\gamma)}}}\left(H(t)-\frac{1}{2}\left(\log _{2} \frac{1+2\left\lfloor N^{1-\gamma}\right\rfloor}{1+2\left\lfloor(N / 2)^{1-\gamma}\right\rfloor}+\log _{2} \frac{\mathrm{E} V_{N / 2}}{\mathrm{E} V_{N}}\right)\right) \rightarrow 0 .
$$


It follows from Lemma 3.4 that

$$
\frac{\ln 2}{\sqrt{C_{10} N^{-(1-\gamma)}}}\left(\log _{2} \frac{V_{N}}{\mathrm{E} V_{N}}-\log _{2} \frac{V_{N / 2}}{\mathrm{E} V_{N / 2}}\right) \stackrel{\mathrm{D}}{\rightarrow} N(0,1),
$$

which completes the result.

The condition that $4 \beta>(4 H(t)-3)(1-\gamma)$ is always satisfied for $H(t) \leq \frac{3}{4}$. For all functions $H(t), \gamma$ can be chosen such that $4 \beta>(4 H(t)-3)(1-\gamma)$.

\section{Appendix A.}

Let $\phi$ be a Schwartz function. Then

$$
\begin{aligned}
\left\langle W_{H}(t), \operatorname{Re} \int_{\mathbb{R}} \phi(\xi) \tilde{W}(\mathrm{~d} \xi)\right\rangle & =\frac{\mathrm{d}}{\mathrm{d} t}\left\langle B_{H}(t), \operatorname{Re} \int_{\mathbb{R}} \phi(\xi) \tilde{W}(\mathrm{~d} \xi)\right\rangle \\
& =\frac{\mathrm{d}}{\mathrm{d} t} \operatorname{Re} \int_{\mathbb{R}} \frac{\mathrm{e}^{\mathrm{i} \xi t}-1}{|\xi|^{H+1 / 2}} \overline{\phi(\xi)} \mathrm{d} \xi \\
& =\operatorname{Re} \int_{\mathbb{R}} \frac{\mathrm{i} \xi \mathrm{e}^{\mathrm{i} \xi t}}{|\xi|^{H+1 / 2}} \overline{\phi(\xi)} \mathrm{d} \xi
\end{aligned}
$$

and, so,

$$
\left\langle\int_{0}^{t} W_{H(s)}(s) \mathrm{d} s, \operatorname{Re} \int_{\mathbb{R}} \phi(\xi) \tilde{W}(\mathrm{~d} \xi)\right\rangle=\left\langle Y_{H}(t), \operatorname{Re} \int_{\mathbb{R}} \phi(\xi) \tilde{W}(\mathrm{~d} \xi)\right\rangle,
$$

which proves that (2.1) and (2.2) are equal.

Lemma A.1. Let $f \in C^{2}([a, b])$ and let $c_{1}, c_{2}>0$ be constants. Then, for any $N, r, s, x, y$, and $z$ satisfying $0<|z|<1<N,|z|^{s} \leq c_{1}$, $(\ln |z|)(|x|+|y|) \leq c_{2},|x|,|y| \leq C_{1} N^{-\beta}$, and $r+i x+j y \in[a, b]$ for $i=0,1$ and $j=0,1$, there exists a constant $K>0$, depending only on $f, c_{1}, c_{2}, \beta$, and $C_{1}$, such that

$$
\left.\left|\sum_{i=0}^{1} \sum_{j=0}^{1}(-1)^{i+j} f(r+i x+j y)\right| z\right|^{s+i x+j y} \mid \leq K(1+\ln |z|)^{2} N^{-2 \beta} .
$$

The result is established by expanding in Taylor series.

Proof of Theorem 2.1. Begin by approximating $H(t)$ on a $2^{-n}$ grid; then

$$
H_{n}(s)=\sum_{i} H\left(i / 2^{n}\right) \mathbf{1}_{\left[i / 2^{n},(i+1) / 2^{n}\right)}(s),
$$

and we let

$$
Y_{n}(t)=\int_{\mathbb{R}}\left(\int_{0}^{t} \frac{\mathrm{i} \xi \mathrm{e}^{\mathrm{i} \xi s}}{|\xi|^{H_{n}(s)+1 / 2}} \mathrm{~d} s\right) \tilde{W}(\mathrm{~d} \xi)
$$


Since $Y_{n}$ is the sum of increments of fractional Brownian motion we can estimate that

$$
\begin{aligned}
\mathrm{E} Y_{n}(t)^{2}= & \sum_{i=1}^{\left\lfloor 2^{n} t\right\rfloor} \sum_{j=1}^{\left\lfloor 2^{n} t\right\rfloor} A\left(\frac{H\left(i / 2^{n}, j / 2^{n}\right)}{2}\right) \frac{1}{2} 2^{-n\left(H\left(i / 2^{n}, j / 2^{n}\right)\right)} \\
& \times\left[|i-j+1|^{H\left(i / 2^{n}, j / 2^{n}\right)}-2|i-j|^{H\left(i / 2^{n}\right)+H\left(j / 2^{n}\right)}\right. \\
& \left.+|i-j-1|^{H\left(i / 2^{n}\right)+H\left(j / 2^{n}\right)}\right] \\
= & \left\{\sum_{i=1}^{\left\lfloor 2^{n} t\right\rfloor} \sum_{j=1}^{\left\lfloor 2^{n} t\right\rfloor} A\left(H\left(\frac{i}{2^{n}}\right)\right) \frac{1}{2} 2^{-n 2 H\left(i / 2^{n}\right)} \phi\left(i-j, 2 H\left(\frac{i}{2^{n}}\right)\right)\right. \\
& \left.+\sum_{i=1}^{\left\lfloor 2^{n} t\right\rfloor} \sum_{j=1}^{\left\lfloor 2^{n} t\right\rfloor} A\left(\frac{H\left(i / 2^{n}, j / 2^{n}\right)}{2}\right) \frac{1}{2} 2^{-n\left(H\left(i / 2^{n}, j / 2^{n}\right)\right)} \phi\left(i-j, H\left(\frac{i}{2^{n}}, \frac{j}{2^{n}}\right)\right)\right\} \\
& +\left\{-A\left(H\left(\frac{i}{2^{n}}\right)\right) \frac{1}{4} 2^{-n 2 H\left(i / 2^{n}\right)} \phi\left(i-j, 2 H\left(\frac{i}{2^{n}}\right)\right)\right. \\
= & \quad-A(H\}+\{I I\}, \\
& \left.\left.\left\{\frac{j}{2^{n}}\right)\right) \frac{1}{4} 2^{-n 2 H\left(j / 2^{n}\right)} \phi\left(i-j, 2 H\left(\frac{j}{2^{n}}\right)\right)\right\}
\end{aligned}
$$

where $\phi(i, \eta)=|i+1|^{\eta}-2|i|^{\eta}+|i-1|^{\eta}$. By the dominated convergence theorem with dominating function $K\left[A(H(s)) H(s) s^{2 H(s)-1}+A(H(s)) H(s)(1-s)^{2 H(s)-1}\right]$, we find that

$$
\begin{aligned}
I & =\sum_{i=1}^{\left\lfloor 2^{n} t\right\rfloor} \sum_{j=1}^{\left\lfloor 2^{n} t\right\rfloor} A\left(H\left(\frac{i}{2^{n}}\right)\right) \frac{1}{2} 2^{-n 2 H\left(i / 2^{n}\right)} \phi\left(i-j, 2 H\left(\frac{i}{2^{n}}\right)\right) \\
& \rightarrow \int_{0}^{t} A(H(s)) H(s) s^{2 H(s)-1} \mathrm{~d} s+\int_{0}^{t} A(H(s)) H(s)(1-s)^{2 H(s)-1} \mathrm{~d} s
\end{aligned}
$$

as $n \rightarrow \infty$. For large $i, \phi(i, \eta) \approx \eta(\eta-1)|i|^{\eta-2}$. By Lemma A.1, for any $\alpha$ with $-1<\alpha<$ $2 a+2 \beta-2$, there exists a $C_{2}$ such that

$$
\left|f_{H}(x, y)\right| \leq C_{2} \min \{|x-y|, 1\}^{\alpha} .
$$

In particular, this implies that $f_{H}(x, y) \in L^{1}\left([0, t]^{2}\right)$. Another application of the dominated convergence theorem, with dominating function $K f_{H}(x, y)$, shows that

$$
I I \rightarrow \frac{1}{4} \int_{0}^{t} \int_{0}^{t} f_{H}(x, y) \mathrm{d} x \mathrm{~d} y .
$$

Similar calculations in estimating $\mathrm{E}\left(Y_{n}-Y_{m}\right)^{2}$ show that $Y_{n}$ is a Cauchy sequence in $L^{2}$. Finally, the pointwise convergence

$$
\int_{0}^{t} \frac{\mathrm{i} \xi \mathrm{e}^{\mathrm{i} \xi s}}{|\xi|^{H_{n}(s)+1 / 2}} \mathrm{~d} s \rightarrow \int_{0}^{t} \frac{\mathrm{i} \xi \mathrm{e}^{\mathrm{i} \xi s}}{|\xi|^{H(s)+1 / 2}} \mathrm{~d} s
$$

implies that $Y_{n} \rightarrow Y$ in $L^{2}$. 
It is sufficient to show local asymptotic self-similarity at 0 and, since integrated fractional white noise is a zero-mean Gaussian process, it is sufficient to show that, for each $s$ and $t$,

$$
\mathrm{E} h^{-2 H(0)} Y_{H}(s h) Y_{H}(t h) \rightarrow A(H(0)) \mathrm{E} B_{H(0)}(s) B_{H(0)}(t) .
$$

Estimating using (2.5) and (A.1), it follows that

$$
\mathrm{E} h^{-2 H\left(t_{0}\right)} Y_{H}(s h)^{2} \rightarrow A\left(H\left(t_{0}\right)\right) s^{2 H\left(t_{0}\right)}=A(H(0)) \mathrm{E} B_{H(0)}(s)^{2}
$$

and, writing $2 Y_{H}(s h) Y_{H}(t h)=2 Y_{H}(s h)^{2}+2 Y_{H}(t h)^{2}-\left(Y_{H}(s h)-Y_{H}(t h)\right)^{2}$, (A.2) follows.

Continuity and the Hölder exponent follow from the application of Kolmogorov's continuity theorem.

Altering the proof of Theorem 2.1, we obtain the following corollary.

Corollary A.1. For $t_{1}<t_{2} \leq t_{3}<t_{4}$, the expected value of $\left(Y\left(t_{2}\right)-Y\left(t_{1}\right)\right)\left(Y\left(t_{4}\right)-Y\left(t_{3}\right)\right)$ is given by

$$
\frac{1}{2} \int_{t_{1}}^{t_{2}} \int_{t_{3}}^{t_{4}} A\left(\frac{H(x, y)}{2}\right) H(x, y)(H(x, y)-1)|x-y|^{H(x, y)-2} \mathrm{~d} x \mathrm{~d} y .
$$

Proof of Lemma 3.1. Let $a_{N}=\min \left\{H(t): s \in\left[t-N^{-\gamma}, t+N^{-\gamma}\right]\right\}, b_{N}=\max \{H(t)$ : $\left.s \in\left[t-N^{-\gamma}, t+N^{-\gamma}\right]\right\}, A_{\min }=\min \left\{A(H(t)): s \in\left[t-N^{-\gamma}, t+N^{-\gamma}\right]\right\}$, and $A_{\max }=$ $\max \left\{A(H(t)): s \in\left[t-N^{-\gamma}, t+N^{-\gamma}\right]\right\}$. By the Hölder condition on $H(t),\left|a_{N}-H(t)\right| \leq$ $C_{1} N^{-\gamma \beta}$ and $\left|A_{\min }-A(H(t))\right| \leq c N^{-\beta}$. Taking $N$ large enough that $2 H(t) \leq 2 b_{N}<$ $2 H(t)+\gamma \beta<\alpha+2$, from Theorem 2.1 and (A.1) we have

$$
\begin{aligned}
\mathrm{E}\left(X_{i+1}-X_{i}\right)^{2} & =\mathrm{E}\left(2 X_{i+1}^{2}+2 X_{i}^{2}-\left(Y_{H}\left(t+\frac{j+1}{N}\right)-Y_{H}\left(t+\frac{j-1}{N}\right)\right)^{2}\right) \\
& \leq 4 A_{\max } N^{-2 a_{N}}-A_{\min }(N / 2)^{-2 b_{N}}+C_{2} N^{-\alpha-2}
\end{aligned}
$$

Since $\left|A_{\max }-A(H(t))\right| \leq c N^{-\gamma \beta}$ and $\left|N^{2 H(t)-2 a_{N}}-1\right| \leq c \ln (N) N^{-\gamma \beta}$,

$$
\mathrm{E}\left(X_{i+1}-X_{i}\right)^{2}-\left(4-4^{H(t)}\right) A(H(t)) N^{-2 H(t)} \leq C_{3} \ln (N) N^{-2 H(t)-\gamma \beta} .
$$

The reverse inequality similarly holds, proving (3.1). Now, for $|i-j|>2$ we define $I$ as

$$
\begin{aligned}
\int_{(t+i) / N}^{t+(i+1) / N} \int_{t+j / N}^{t+(j+1) / N} \mid \sum_{k=0}^{1} \sum_{\ell=0}^{1} & (-1)^{k+\ell} A\left(\frac{H(x-k / N, y-\ell / N)}{2}\right) H\left(x-\frac{k}{N}, y-\frac{\ell}{N}\right) \\
& \times\left(H\left(x-\frac{k}{N}, y-\frac{\ell}{N}\right)-1\right) \\
& \times\left|\frac{i-j}{N}\right|^{H(x-k / N, y-\ell / N)-2} \mid \mathrm{d} x \mathrm{~d} y .
\end{aligned}
$$


Then, by Lemma A.1,

$$
\begin{aligned}
|I| \leq|i-j|^{2 H(t)-2} N^{-2 H(t)} & \\
\times \int_{0}^{1} \int_{0}^{1} \sum_{k=0}^{1} \sum_{\ell=0}^{1} \mid & A\left(\frac{H(t+(x-k) / N, t+(y-l) / N)}{2}\right) H\left(t+\frac{x-k}{N}, t+\frac{y-l}{N}\right) \\
\times & \left(H\left(t+\frac{x-k}{N}, t+\frac{y-l}{N}\right)-1\right) \\
\times & \left|\frac{i-j}{N}\right|^{H(t+(x-k) / N, t+(y-l) / N)-2 H(t)} \mid \\
\times & N^{-H(t+(x-k) / N, t+(y-l) / N)} \mathrm{d} x \mathrm{~d} y \\
\leq C_{4} \ln (|i-j|)^{2} \mid i- & \left.j\right|^{2 H(t)-2} N^{-2 H(t)-2 \beta} .
\end{aligned}
$$

Let

$$
\begin{aligned}
I I=\int_{(t+i) / N}^{t+(i+1) / N} & \int_{t+j / N}^{t+(j+1) / N}\left[\sum_{k=0}^{1} \sum_{\ell=0}^{1} A\left(\frac{H(x-k / N, y-l / N)}{2}\right)\right. \\
& \times H\left(x-\frac{k}{N}, y-\frac{l}{N}\right)\left(H\left(x-\frac{k}{N}, y-\frac{l}{N}\right)-1\right) \\
& \times\left(\left|\frac{i-j}{N}\right|^{H(x-k / N, y-l / N)-2}-\left|\left(x-\frac{k}{N}\right)-\left(y-\frac{l}{N}\right)\right|^{H(x-k / N, y-l / N)-2}\right) \\
-\sum_{k=0}^{1} & \sum_{\ell=0}^{1} A(H(t)) 2 H(t)(2 H(t)-1) \\
& \left.\times\left(\left|\frac{i-j}{N}\right|^{2 H(t)-2}-\left|\left(x-\frac{k}{N}\right)-\left(y-\frac{l}{N}\right)\right|^{2 H(t)-2}\right)\right] \mathrm{d} y \mathrm{~d} x .
\end{aligned}
$$

Then

$$
\begin{aligned}
|I I| \leq \int_{t+(i-1) / N}^{t+(i+1) / N} \int_{t+(j-1) N}^{t+(j+1) / N} \mid & A\left(\frac{H(x, y)}{2}\right) H(x, y)(H(x, y)-1) \\
& \times\left(\left|\frac{i-j}{N}\right|^{H(x, y)-2}-|x-y|^{H(x, y)-2}\right) \\
& -A(H(t)) 2 H(t)(2 H(t)-1) \\
& \times\left(\left|\frac{i-j}{N}\right|^{2 H(t)-2}-|x-y|^{2 H(t)-2}\right) \mid \mathrm{d} y \mathrm{~d} x .
\end{aligned}
$$

If

$$
(x, y) \in\left[t+\frac{i-1}{N}, t+\frac{i+1}{N}\right] \times\left[t+\frac{j-1}{N}, t+\frac{j+1}{N}\right] \quad \text { and } \quad|i-j|>2,
$$


then $|i-j|-2 \leq N|x-y| \leq|i-j|+2$ and, so,

$$
\left|\ln \frac{|i-j|}{N}-\ln \right| x-y|| \leq 4|i-j|^{-1} .
$$

It follows that

$$
\left.|| \frac{i-j}{N}\right|^{2 H(t)-2}-|x-y|^{2 H(t)-2}|\leq| i-\left.j\right|^{2 H(t)-3} N^{-2 H(t)+2} .
$$

Thus,

$$
\begin{aligned}
& \mid A\left(\frac{H(x, y)}{2}\right) H(x, y)(H(x, y)-1)\left(\left|\frac{i-j}{N}\right|^{H(x, y)-2}-|x-y|^{H(x, y)-2}\right) \\
& -A(H(t)) 2 H(t)(2 H(t)-1)\left(\left|\frac{i-j}{N}\right|^{2 H(t)-2}-|x-y|^{2 H(t)-2}\right) \mid \\
& \leq\left|A\left(\frac{H(x, y)}{2}\right) H(x, y)(H(x, y)-1)-A(H(t)) 2 H(t)(2 H(t)-1)\right| \\
& \quad \times\left|\frac{i-j}{N}\right|^{2 H(t)-2}-|x-y|^{2 H(t)-2} \mid \\
& \quad+A\left(\frac{H(x, y)}{2}\right) H(x, y)(H(x, y)-1)\left|\frac{i-j}{N}\right|^{2 H(t)-2} \\
& \quad \times\left(\left(1-\left(\frac{N|x-y|}{|i-j|}\right)^{2 H(t)-2}\right)\left(1-|x-y|^{H(x, y)-2 H(t)}\right)\right. \\
& \left.\quad+\left(\left|\frac{i-j}{N}\right|^{H(x, y)-2 H(t)}-|x-y|^{H(x, y)-2 H(t)}\right)\right) \\
& \leq C_{5} \ln (|i-j|)^{2}|i-j|^{2 H(t)-3} N^{2-2 H(t)-\gamma \beta},
\end{aligned}
$$

by expanding out in Taylor series. Hence,

$$
|I I| \leq C_{5} \ln (|i-j|)^{2}|i-j|^{2 H(t)-3} N^{-2 H(t)-\gamma \beta} .
$$

Adding (A.3) and (A.4) and using Corollary A.1 proves (3.2) for $|i-j|>2$. The case in which $|i-j| \leq 2$ is proved similarly to (3.1).

\section{Acknowledgement}

I would like to thank my supervisor, Chris Heyde, for his advice and guidance while carrying out this research, which was done at the Australian National University.

\section{References}

[1] Anh, V. V., Yu, Z. G., Wanliss, J. A. and Watson, S. M. (2005). Prediction of magnetic storm events using the $D_{s t}$ index. Nonlinear Process. Geophys. 12, 799-806.

[2] Ayache, A. And Lévy VéHel, J. (2004). On the identification of the pointwise Hölder exponent of the generalized multifractional Brownian motion. Stoch. Process. Appl. 111, 119-156.

[3] Ayache, A., Benassi, A., Cohen, S. And Lévy VéHel, J. (2005). Regularity and identification of generalized multifractional Gaussian processes. In Séminaire de Probabilités XXXVIII (Lecture Notes Math. 1857), Springer, Berlin, pp. 290-312. 
[4] Benassi, A., Cohen, S. AND Istas, J. (1998). Identifying the multifractional function of a Gaussian process. Statist. Prob. Lett. 39, 337-345.

[5] Benassi, A., Bertrand, P., Cohen, S. and Istas, J. (1999). Identification d'un processus gaussien multifractionnaire avec des ruptures sur la fonction d'échelle. C. R. Acad. Sci. Paris Sér. I Math. 329, 435-440.

[6] Benassi, A., Bertrand, P., Cohen, S. And Istas, J. (2000). Identification of the Hurst index of a step fractional Brownian motion. Statist. Infer. Stoch. Process. 3, 101-111.

[7] Carbone, A., Castelli, G. and Stanley, H. E. (2004). Time-dependent Hurst exponent. Physica A 344, 267-271.

[8] Chung, K. L. (2001). A Course in Probability Theory. Academic Press, San Diego, CA.

[9] Elliott, R. J. And VAn der Hoek, J. (2003). A general fractional white noise theory and applications to finance. Math. Finance 13, 301-330.

[10] Janson, S. (1997). Gaussian Hilbert Spaces. Cambridge University Press.

[11] Mandelbrot, B. B. and Fisher, A. and Calvet, L. (1997). A multifractal model of asset returns. CFDP 1164. Available at http://cowles.econ.yale.edu/P/cd/dy1997.htm.

[12] Murali Krishna, P., Gadre, V. M. And Desai, V. B. (2003). Multifractal Based Network Traffic Modeling. Kluwer, Boston, MA.

[13] Peltier, R. ANd LÉvy Véhel, J. (1995). Multifractional Brownian motion: definition and preliminary results. Res. Rept. 2645, INRIA.

[14] Sreenivasan, K. R. (1991). Fractals and multifractals in fluid turbulence. Ann. Rev. Fluid Mech. 23, 539-600.

[15] Stoev, S. A. And Taqqu, M. S. (2006). How rich is the class of multifractional Brownian motions? Stoch. Process. Appl. 116, 200-221.

[16] Watkins, N. W. et al. (2005). Towards synthesis of solar wind and geomagnetic scaling exponents: a fractional Lévy motion model. Space Sci. Rev. 121, 271-284.

[17] YU, Z.-G., ANH, V. AND LAU, K.-S. (2003). Multifractal and correlation analyses of protein sequences from complete genomes. Phys. Rev. E 68, 021913. 